\begin{tabular}{|c|l|}
\hline Title & A ttenuation and dispersion of antiplane shear waves due to scattering by many two-dimensional cavities \\
\hline Author(s) & Kawahara, Jun; Ohno, Taichi; Y omogida, Kiy oshi \\
\hline Citation & $\begin{array}{l}\text { Journal of the A coustical Society of A merica, 125(6), 3589-3596 } \\
\text { https://doi.org/10.1121/1.3124779 }\end{array}$ \\
\hline Issue Date & 2009-06 \\
\hline Doc URL & http://hdl.handle.net/2115/40200 \\
\hline Rights & $\begin{array}{l}\text { Copyright 2009 A coustical Society of A merica. This article may be downloaded for personal use only. Any other use } \\
\text { requires prior permission of the author and the A coustical Society of A merica. The following article appeared in J. } \\
\text { A coust. Soc. Am., 125(6), pp. 3589-3596 and may be found at http:/dx.doi.org/10.1121/1.3124779 }\end{array}$ \\
\hline Type & article \\
\hline File Information & JA SA 125-6_p3589-3596.pdf \\
\hline
\end{tabular}

Instructions for use 


\title{
Attenuation and dispersion of antiplane shear waves due to scattering by many two-dimensional cavities
}

\author{
Jun Kawahara ${ }^{a)}$ \\ Faculty of Science, Ibaraki University, 2-1-1 Bunkyo, Mito 310-8512, Japan \\ Taichi Ohno b) \\ Graduate School of Science and Engineering, Ibaraki University, 2-1-1 Bunkyo, Mito 310-8512, Japan \\ Kiyoshi Yomogida \\ Graduate School of Science, Hokkaido University, North 10 West 8, Kita-ku, Sapporo 060-0810, Japan
}

(Received 13 March 2008; revised 9 January 2009; accepted 1 April 2009)

\begin{abstract}
Propagation and scattering of antiplane shear waves within media with two-dimensional cavities are numerically simulated, and the attenuation and phase velocities are experimentally determined. The results are compared with the predictions by the Foldy theory and its three corrected versions. If the cavity concentrations are small such as 0.02 , the differences among the theoretical predictions are insignificant, and every theory is consistent with the experimental results. For higher concentrations such as 0.1 , the differences become significant, but there are no objective grounds to say that any of the corrected versions of the Foldy theory works better than the original. If the error tolerance is as high as $10 \%$, the simple Foldy formula may remain useful for concentrations up to about 0.1 . (C) 2009 Acoustical Society of America. [DOI: 10.1121/1.3124779]
\end{abstract}

PACS number(s): 43.35.Cg, 43.20.Jr, 43.20.Gp, 43.20.Bi [RAS] Pages: 3589-3596

\section{INTRODUCTION}

Wave propagation in a medium with distributed scatterers results in the attenuation and dispersion of coherent wave fields, even if each constituent is lossless. This phenomenon has been studied in a variety of research areas because of its wide applications. Examples are acoustic scattering in the ocean and in biological media, ${ }^{1}$ ultrasonic nondestructive evaluation of materials, ${ }^{2}$ and seismic scattering in the earth's lithosphere. ${ }^{3}$ Many of such researches have been based on theoretical modeling or laboratory experiments as stated below. In contrast, the purpose of the present article is to approach the phenomenon by the use of a numerical method of wave simulations. Because of computational tractability, only a specific case of antiplane shear ( $\mathrm{SH}$ ) wave scattering due to two-dimensional (2D) cavities is treated, which enables the authors to achieve simulations with high accuracy at relatively low computational costs.

A large variety of theories has been proposed for the problem stated above. Among others, Foldy ${ }^{4}$ established a category of theories (multiple scattering theories) about 60 years ago. He considered scalar wave propagation in media with randomly distributed three-dimensional (3D) isotropic scatterers and derived the following well-known formula:

$$
K^{2}=k^{2}+4 \pi n f,
$$

in which $K$ is the effective wavenumber of mean (ensembleaveraged) wave fields, $k$ is the wavenumber in the absence of scatterers, $n$ is the number density of the scatterers, and $f$ is

\footnotetext{
a) Author to whom correspondence should be addressed. Electronic mail: junk@mx.ibaraki.ac.jp

${ }^{b}$ Present address: OYO RMS Corp., 3-11-15 Akasaka, Minato-ku, Tokyo 107-0052, Japan.
}

the isotropic scattering amplitude of a single scatterer. This equation gives the solution $K$ as a function of $k$, whose real and imaginary parts correspond to the phase velocity and attenuation, respectively. Foldy's theory is based on the assumption that the scatterers are distributed very sparsely and independently so that its validity is limited to sufficiently small $n$. Later, Lax ${ }^{5}$ verified that Eq. (1) remains valid for anisotropic scatterers if $f$ is replaced with the forward scattering amplitude $f(0)$ :

$$
K^{2}=k^{2}+4 \pi n f(0) \text {. }
$$

Many researchers tried to improve the theory in order that it may be valid for denser scatterer distributions by taking account of the multiple scattering more appropriately. Lax $^{6}$ proposed the quasicrystalline approximation, in which statistical information on the relative location of two scatterers is described by a pair-correlation function. Waterman and Truell $^{7}$ derived a formula alternative to Eq. (2) under the condition of non-overlapping scatterers with infinitesimal volumes. Despite its lasting popularity, the Waterman-Truell formula was shown to be incorrect by Lloyd and Berry, ${ }^{8}$ who presented an alternative one. Other researchers applied more appropriate pair-correlation functions for scatterers with finite sizes. ${ }^{9-12}$ Still other researchers proposed corrected versions of Foldy's theory not based on the quasicrystalline approximation. Examples are the works based on a diagram method $^{13,14}$ and the combinations of the Waterman-Truell formula and a self-consistent method. ${ }^{15}$ Concerning other categories of theories on wave propagation in inhomogeneous media (such as self-consistent methods), see Ref. 16 and the references therein.

The above-mentioned theories, including Foldy's, have been exploited in order to describe the results of laboratory experiments on wave transmission in bubbly liquids, suspen- 
sions, emulsions, particulate composites, and materials with cavities (see Refs. 11, 12, and 16-23 and the references therein). The predictions of all such theories coincide with each other up to the first order of $n$, and they are consistent with the experimental data for small volume concentrations of scatterers. ${ }^{17,20}$ The limits of the concentrations below which the theories remain valid, however, vary among studies. One possible reason for this may be the factors that are not considered in the theories but essential in the experiments, such as viscous and thermal dissipation ${ }^{20}$ and illcontrolled parameters such as scatterer sizes. ${ }^{22}$ On one hand, incorporating such effects appropriately into the theories to bridge this gap is certainly an important task from the practical viewpoint. ${ }^{18-20}$ On the other hand, using computer simulations of wave scattering free from those effects may be helpful in examining the validity of the theories more directly; though less practical than laboratory ones, numerical experiments could be configured more loyally to the assumptions made in the theories. ${ }^{24-27}$ Another possible reason for the discrepancy among the experimental results may be the variation in the methods to measure the attenuation and phase velocities of coherent waves. Some researchers measured the amplitude changes and moving speeds of the peaks of transmitting pulses, ${ }^{22-24}$ whereas others calculated their spectral changes. ${ }^{12,17-21}$ Although the latter method has the advantage of high spectral resolution, the estimates thus obtained could be affected by the incoherent waves included within the pulses. Ensemble averaging of the waveform data was shown to be effective in reducing the incoherent waves as well as noises. ${ }^{12,17,19,27}$

In this article, multiple scattering theories of Foldy, ${ }^{4,5}$ Waterman and Truell, ${ }^{7}$ Lloyd and Berry, ${ }^{8}$ and Ye and Ding ${ }^{13}$ are compared with numerical-experimentally measured attenuation and phase velocities. To focus on the validity of the theories, numerical experiments are performed as mentioned earlier; antiplane shear wave scattering by 2D circular (or parallel cylindrical) cavities in elastic media are simulated with high accuracy, closely following Benites et al. ${ }^{24}$ The attenuation and phase velocities are measured by applying the spectral ratio technique to averaged waveform data, as was successfully done by Suzuki et al. ${ }^{27}$ Although the model scattering media in the present experiment are rather idealized, it would be a good analog of high-contrast particulate materials and media with small aspect ratio cavities (e.g., cracks).

In Sec. II, the 2D solutions of the above four theories are summarized. In Sec. III, the configuration of the numerical experiments and the data acquisition method are described. The experimentally determined attenuation and dispersion curves are shown in Sec. IV, in which they are compared with the theoretical predictions. The validity of the theories is thereby discussed.

\section{MULTIPLE SCATTERING THEORIES}

All of the theories considered here share the advantage of giving explicit solutions of $K$ like Eq. (2), which in the 2D case are summarized in this section. It is worth noting that all of them are based on a point scatterer assumption. Treating scatterers with finite sizes will be possible by using a proper pair-correlation function, but then the solution $K$ would be obtained only numerically. ${ }^{9-12}$

The 2D version of Foldy's formula (with Lax's correction) (2) can be found in, e.g., Linton and Martin. ${ }^{28}$ It is written in the form

$$
K^{2}=k^{2}-4 \inf (0),
$$

in which $i$ is the imaginary unit. Here $f(\theta)$ is the 2D scattering amplitude for the scattering angle $\theta$ measured from the incident direction.

As stated in Sec. I, Waterman and Truell ${ }^{7}$ took account of the effect of non-overlapping scatterers under the point scatterer assumption. This means that only the "perfect overlapping" of scatterers with finite sizes (or the overlapping of the centers of the scatterers) is excluded. The $2 \mathrm{D}$ version of their formula corresponding to Eq. (3) was given by Angel and Aristégui ${ }^{29}$ as

$$
K^{2}=k^{2}-4 \inf (0)-\frac{4 n^{2}}{k^{2}}\left[f(0)^{2}-f(\pi)^{2}\right] .
$$

Although this formula has been widely used, ${ }^{15-17,21}$ Lloyd and Berry ${ }^{8}$ pointed out that it is based on an incorrect paircorrelation function. According to them, the exclusive volume of a spherical scatterer (whose radius was finally allowed to become zero) actually imposed in the WatermanTruell theory is not spherical but slablike, being perpendicular to the direction of wave propagation. Applying a correct pair-correlation function and taking the limit of point scatterers again, Lloyd and Berry derived an alternative formula, using a technique called the "resummation method" coming from nuclear physics. However, the Lloyd-Berry formula seems less popular than the Waterman-Truell formula. Linton and Martin ${ }^{30}$ inferred that it is perhaps because the Lloyd-Berry formula is less simpler and its derivation seems suspect. Nevertheless, they obtained a new and clearer derivation of this formula. Linton and Martin $^{28}$ also developed its $2 \mathrm{D}$ version as

$$
K^{2}=k^{2}-4 \inf (0)+\frac{8 n^{2}}{\pi k^{2}} \int_{0}^{\pi} \cot (\theta / 2) \frac{d}{d \theta} f(\theta)^{2} d \theta .
$$

It has been clarified that Foldy's approach is equivalent to taking into account "successive scattering" only, whose paths do not touch any scatterers more than once. ${ }^{1,13}$ Using a diagram method, Ye and Ding ${ }^{13}$ incorporated into formula (1) the effect of triple scattering involving two scatterers, as depicted in Fig. 1. The result is that

$$
K^{2}=k^{2}+4 \pi n(f+\Delta f),
$$

in which $\Delta f$ is the variation in the scattering amplitude of $\mathrm{S} 1$ due to the incidence of waves that are scattered by S1 and then scattered back by $\mathrm{S} 2$. This quantity is given by

$$
\Delta f=n f^{3} \iiint\left[\frac{e^{i k r}}{r}\right]^{2} d \mathbf{r}=\frac{2 \pi i n f^{3}}{k} .
$$

The $2 \mathrm{D}$ version of these equations may be inferred by replacing the term $f \exp (i k r) / r$, which represents a far-field singlescattered wave, by its 2D counterpart $f H_{0}^{(1)}(k r)$, in which 


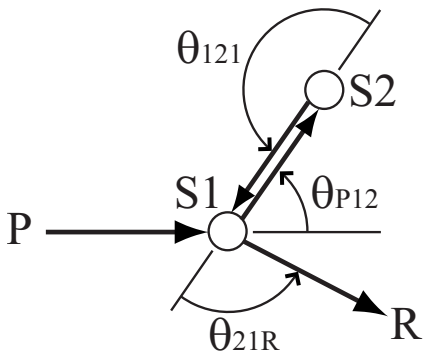

FIG. 1. Triple scattering involving two scatterers S1 and S2. Here P and R denote the source and receiver, respectively.

$H_{0}^{(1)}()$ is the Hankel function of the first kind. The result in the $2 \mathrm{D}$ case is that

$$
\begin{aligned}
& K^{2}=k^{2}-4 i n(f+\Delta f), \\
& \Delta f=n f^{3} \iint\left[H_{0}^{(1)}(k r)\right]^{2} d \mathbf{r}=\frac{4 n f^{3}}{\pi k^{2}} .
\end{aligned}
$$

The extension of these relations to the case of anisotropic scattering may be achieved as follows. To be consistent with formula (3) to which only the forward scattering $(\theta=0)$ contributes, it may be enough to consider only the contribution of the triple scattering depicted in Fig. 1 with the directions $\mathrm{P} \rightarrow \mathrm{S} 1$ and $\mathrm{S} 1 \rightarrow \mathrm{R}$ being the same. One can therefore let $\theta_{21 \mathrm{R}}=\pi-\theta_{\mathrm{P} 12}$. Moreover, the second scattering is always backward, that is, $\theta_{121}=\pi$. Thus the Ye-Ding formula in the case of 2D anisotropic scattering may be inferred as

$$
K^{2}=k^{2}-4 i n\left[f(0)+\frac{4 n f(\pi)}{\pi^{2} k^{2}} \int_{0}^{\pi} f(\theta) f(\pi-\theta) d \theta\right] .
$$

Note that Henyey ${ }^{14}$ pointed out that the Ye-Ding formula may overestimate the contribution of the triple scattering of the above type because it neglects the scattering attenuation during the first and second scattering processes. His corrected formula for $3 \mathrm{D}$ isotropic scattering, resulting in a cubic equation with respect to $K$, would give results falling between those by the theories of Foldy and Ye-Ding. The extension of the formula, however, to anisotropic scattering is not straightforward and hence it is not considered here.

For $K=K(k)$ given by any of the theories, the attenuation coefficient $\alpha$ and the phase velocity $c$ of the mean (or coherent) waves are evaluated by the relations

$$
\alpha=\operatorname{Im} K, \quad \frac{c}{c_{0}}=\frac{k}{\operatorname{Re} K},
$$

in which "Im" and "Re" denote the imaginary and real parts, respectively, and $c_{0}$ is the wave speed of a matrix surrounding scatterers. In the case of a cylindrical cavity with the radius $a, f(\theta)$ is expressed as the sum of an infinite series with the Bessel and Hankel functions ${ }^{31}$

$$
f(\theta)=-\sum_{m=0}^{\infty} \frac{J_{m-1}(k a)-J_{m+1}(k a)}{H_{m-1}^{(1)}(k a)-H_{m+1}^{(1)}(k a)} \epsilon_{m} \cos m \theta,
$$

in which $\epsilon_{0}=1$ and $\epsilon_{m}=2$ for $m \geqslant 1$.

\section{NUMERICAL EXPERIMENTS}

The configurations of the present numerical experiments follow those of Benites et al., ${ }^{24}$ which are explained below. First, $N$ identical circular cavities are distributed inside a rectangular area with the horizontal size $W$ and vertical one $L$. Here both the cavity diameter $d=2 a$ and the wave speed of the matrix $c_{0}$ are chosen to be unity. It is also assumed that the cavities do not overlap, i.e., they are "impenetrable." Using a uniform random number series, the cavities are distributed inside the area randomly, except for the constraint that the interval between the centers of any pair of cavities must be greater than $1.1 d$. Specifically, the cavity centers $\left(x_{i}, y_{i}\right)$ $(i=1, \ldots, N)$ are randomly generated one by one; if a newly generated center $\left(x_{j}, y_{j}\right)$ does not satisfy the constraint [i.e., if the distance between this and any of the preexisting centers $\left(x_{i}, y_{i}\right)(i<j)$ is smaller than 1.1d], then it is eliminated and another one is newly generated. Here it is also imposed that the distance between any cavity center and the top end of the area must be greater than $d$. Hence the vertical size of the actual distribution area is regarded as $L^{\prime}=L-0.5 d$, and the cavity number density may be defined as $n=N / W L^{\prime}$.

Second, 101 receivers are evenly arrayed along the top end of the area. Note that the distance between any receiver and any cavity boundary is not smaller than $0.5 d$, according to the algorithm of distributing the cavities stated above. A plane Ricker wavelet with the dominant frequency $f_{C}$ is vertically impinged on the bottom end from below, and then waveforms at the receivers are synthesized using the method of Benites et al.; ${ }^{24}$ see their paper as for the source time function and the spectrum of the Ricker wavelet. Although time-domain waveforms are synthesized in the end, the basic calculations are performed in the frequency domain; that is, the responses at various frequencies are computed first, then a time-domain waveform is synthesized from their inverse Fourier transforms with the convolution of a source wavelet (the Ricker wavelet in this case). Note that four ends of the distribution area are transparent with respect to wave transmission. In order to suppress the effect of the scattered waves leaking through the both side ends, the waveforms at $N_{R}$ receivers around the middle of the receiver array are used in the analysis. The value of $N_{R}$ is chosen dependent on the size of the distribution area and the time interval of synthetic waveforms.

Third, the above-mentioned process is repeated for $N_{D}$ different realizations of cavity distributions that are stochastically identical but are generated using different random number series. Then the $N_{R} \times N_{D}$ waveforms obtained are averaged to yield a "synthetic mean wave." This implies the assumption of ergodicity, that is, the equivalence of spatial and ensemble averaging. For sufficiently large $N_{R} \times N_{D}$, incoherent waves would be effectively removed.

Finally, $\alpha$ and $c$ are evaluated from the spectral ratio of the primary parts of the mean wave to the initial wavelet. The time window for the analysis is centered on the arrival time of the central peak of the initial wavelet, with the interval of $4 / f_{C}$; the choice of the interval would not significantly affect the results if the incoherent waves had been sufficiently removed. The evaluation of $\alpha$ and $c$ is conducted for 
TABLE I. Parameters of the cavity distribution models.

\begin{tabular}{cccccccc}
\hline \hline & $N$ & $W / d$ & $L / d$ & $C$ & $N_{R}$ & $N_{D}$ & $\max \left|n f / k^{2}\right|$ \\
\hline Model 1 & 50 & 80 & 30 & 0.017 & 61 & 5 & 0.013 \\
Model 2 & 100 & 80 & 10 & 0.10 & 61 & 10 & 0.078 \\
Model 3 & 100 & 40 & 10 & 0.21 & 41 & 15 & 0.16 \\
\hline
\end{tabular}

the frequency band $0.75 f_{C}-1.5 f_{C}$ and is repeated for different initial wavelets with $f_{C}=0.15,0.3,0.6$, and $1.2\left(\times c_{0} / d\right)$.

The success of the numerical experiments described above depends on the accuracy of the wave simulation method. The method of Benites et al. ${ }^{24}$ adopted here can be categorized into boundary integral methods (or indirect boundary element methods), which probably dates back to Copley $^{32}$ and have been successfully applied to solve various types of elastodynamic problems in engineering and seismology. ${ }^{33-38}$ The method of Benites et al. can accurately simulate wave fields in media with arbitrarily distributed cavities, including anisotropic scattering and every degree of multiple scattering; this is in contrast to the other methods based on the Born approximation or the parabolic one. ${ }^{3,39}$ Note that similar methods were previously applied by Dravinski ${ }^{34}$ and Benites and $\mathrm{Aki}^{36}$ to one or two inclusions. Although the details of the method can be seen in the paper of Benites et al., ${ }^{24}$ the essence is that a scattered wave due to each cavity is expressed as the sum of waves radiated from fictitious line sources distributed behind (but close to) the cavity boundary. More specifically, the sources are evenly spaced along a circle with the radius $0.8 a$, which is concentric with the circular boundary. The source strengths are determined in the least-square sense so as to satisfy the traction-free boundary condition, and thus the wave fields outside the cavities are synthesized. Here the number of the sources per cavity (or the interval between the sources) controls the accuracy of wave simulations. Benites et al. ${ }^{24}$ checked the validity of their method on the basis of three different tests and demonstrated that it really produces sufficiently accurate wave fields if there exist at least four sources per wavelength measured along the cavity boundary (i.e., the number of the sources per cavity is not less than $4 \pi d / \lambda$, in which $\lambda$ is the minimum wavelength considered). Note that this criterion is consistent with the results of other researches on boundary integral methods. ${ }^{33,35,36}$ In the present wave simulations, the number of the sources per cavity is chosen so as to satisfy the criterion. The authors also checked the stability of simulations by temporally increasing the number to large extent in some cases. This turned out not to change the results significantly (within a few percent at most), implying that the original number would be enough.

In Sec. IV, the results for three models of cavity distributions are shown. Table I summarizes the parameters of the models, in which the area concentrations $C=\pi n a^{2}$ are shown instead of $n$. An example of the realizations for each model is depicted in Fig. 2. Model 1, representing the case of sparse distributions, is stochastically identical to the model examined by Benites et al. ${ }^{24}$ In contrast, models 2 and 3 represent the cases of rather dense distributions. Here the denser distributions are realized partly by making the model areas
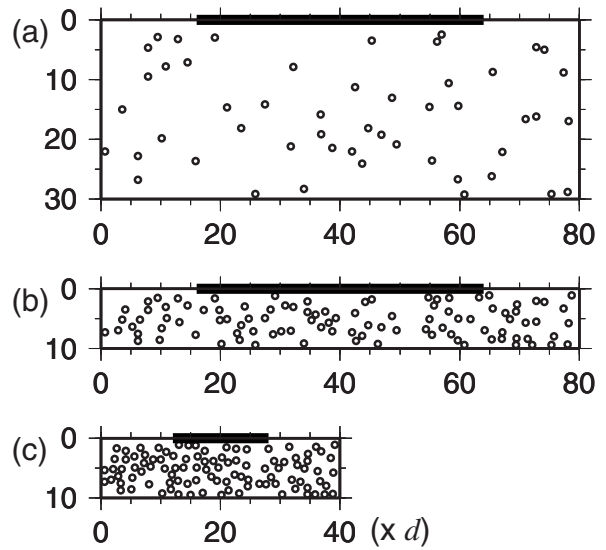

FIG. 2. Examples of the realizations for (a) model 1, (b) model 2, and (c) model 3. The thick bars along the top ends of the model areas designate the receiver arrays.

smaller in order to save the computational costs because the costs strongly depend on the numbers of cavities (more exactly, the numbers of fictitious sources). Note that the $N_{D}$ values listed in Table I were actually determined as a result of repeated evaluation of $\alpha$ and $c$ with the increasing number of the realizations for each model. The repetition was terminated when the addition of a new realization did not significantly change the results anymore, and then the final number of the realizations was defined as $N_{D}$. Consequently, $N_{D}$ is not the same among the models; a smaller model area seems to need more representations for stable ensemble average. Note also that Table I includes the maximum value of $\left|n f(\theta) / k^{2}\right|$ for each model just for reference, which will be discussed later.

The results are to be compared in Sec. IV with the predictions of the theories stated in Sec. II, thus discussing their validity, mainly in terms of the distribution density of cavities. Other parameters that might also limit the validity of the theories will be also discussed there. Here $C$ is employed instead of $n$ as a measure of the distribution density, following the convention in laboratory experiments. ${ }^{11,12,16-23}$ It may be worth mentioning, however, that the use of $C$ may not be effective if the aspect ratios of scatterers are much deviated from unity. An example is distribution of highly oblate cavities (or cracks), for which $C$ would remain negligibly small even for considerably large $n$; there "crack density" $n r^{3}$ ( $r$ is the major axis) is conventionally used instead.

\section{RESULTS AND DISCUSSION}

The synthetic waveforms are exemplified in Fig. 3 in the case of model $1(C=0.017)$ and $f_{C}=0.3$. In Fig. 3(a), the cloud of lines denotes the waveforms at the $N_{R}$ receivers for a model realization. It is clearly illustrated that the waves keep their coherency around the first motion but lose them rapidly, resulting in the incoherent long wave trains composed of the scattered waves. The synthetic mean wave obtained from all of the waveforms is plotted in Fig. 3(b), in which the waveform in the absence of cavities (initial wavelet) is superimposed for comparison. Here the attenuation and delay of the mean wave are clearly recognized. In addition, it is a compact wavelet like the initial one and has no 

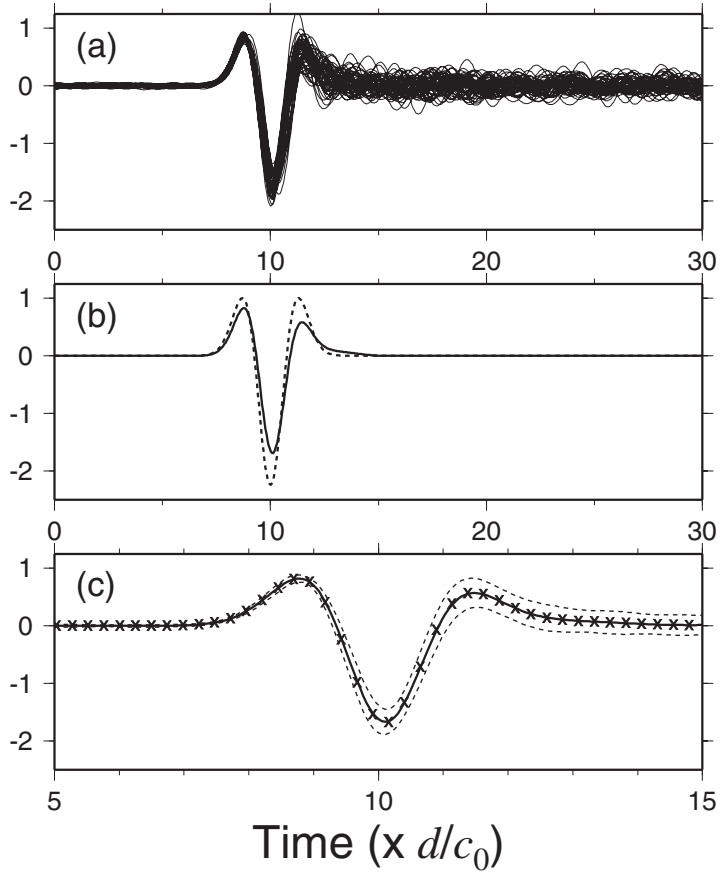

FIG. 3. (a) Synthetic waveforms for model 1 with $f_{C}=0.3$. (b) Synthetic mean wave (solid line) and the initial wavelet (dotted line). (c) Close-up of the primary part. The solid line and the two dotted ones denote the average and the standard deviation of the waveforms. The crosses indicate the mean wave predicted by Foldy formula (3).

long tail. This implies that the present averaging process is successful in removing the incoherent waves, validating the present experimental procedures. In Fig. 3(c), the mean wave predicted by Foldy formula (3) is also plotted; it is completely consistent with the synthetic mean wave. Similar consistency between the synthetic and predicted mean waves is observed also in the other dominant frequencies, suggesting high validity of formula (3) for such sparse scatterer distributions, as expected.

Figure 4 depicts the attenuation and dispersion of the synthetic mean waves for model 1 as functions of the normalized wavenumber $k a$. Here

$$
Q^{-1}=\frac{c}{c_{0}} \frac{2 \alpha}{k}=\frac{2 \operatorname{Im} K}{\operatorname{Re} K}
$$

is plotted instead of $\alpha$ in Eq. (11). Note that the inverse of quality factor $Q^{-1}$, preferably used in seismology, material mechanics, and electric circuits, represents attenuation per wavelength; a small difference in $\alpha$ leads to large one in $Q^{-1}$ at $k a \sim 1$, at which $Q^{-1}$ reaches a peak. Here are plotted the values of $Q^{-1}$ and $c$ determined for $f_{C}=0.15,0.3,0.6$, and 1.2 (corresponding to $k a=0.47,0.94,1.88$, and 3.77, respectively), together with the values predicted by the Foldy formula. Note that the predictions by the other formulas are not shown because the deviations from those by the Foldy formula are insignificant in the present case. The experimental values are closely followed by the predicted ones on the whole, as expected again. There exist, however, minor systematic discrepancies on $Q^{-1}$ in the range $2<k a<3$. As will be discussed later, these discrepancies might be due to the effect of finite scatterer sizes.

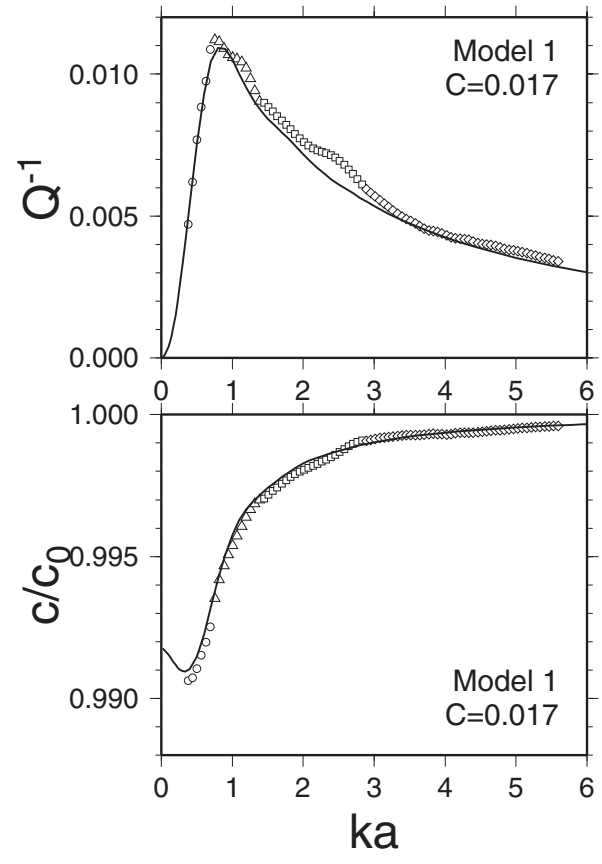

FIG. 4. Attenuation and dispersion curves for model 1. The open symbols with different shapes denote the experimental values for different $f_{C}$. The solid lines denote the values predicted by Foldy formula (3).

Figure 5 shows $Q^{-1}$ and $c$ obtained for model $2(C$ $=0.10)$ and for $f_{C}=0.15,0.3$, and 0.6 ; the case of $f_{C}=1.2$ is omitted because of high computational costs. Now the predictions by all of the four theories are depicted. The differences among the theoretical values are remarkable in the present case, especially on $Q^{-1}$ for $k a \sim 1$ and $c$ for $k a<1$. In the latter wavenumber range, the formulas of Lloyd-Berry (5) and Ye-Ding (10) give very close $c$ values, between

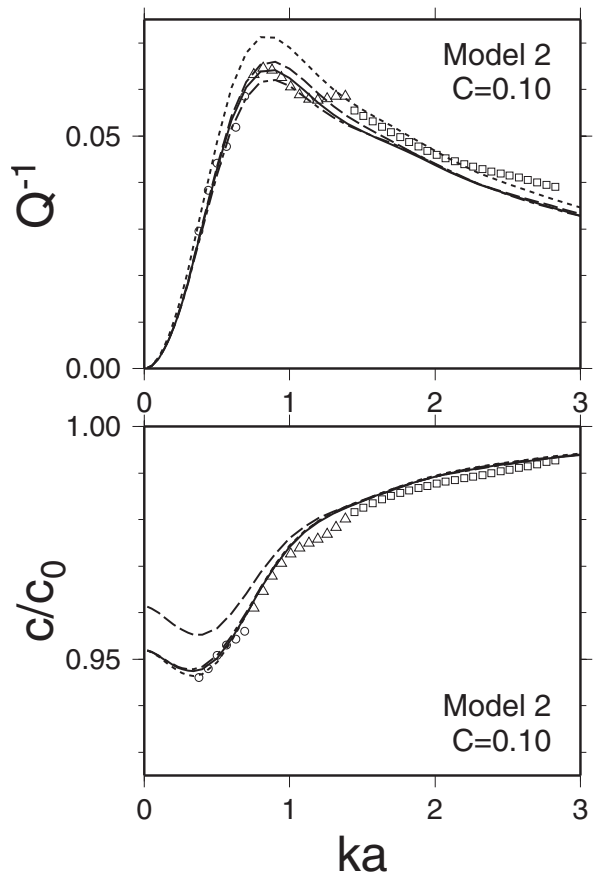

FIG. 5. Attenuation and dispersion curves for model 2. The open symbols denote the experimental values. The solid, dashed, dotted, and dash-dotted lines denote the predictions by the theories of Foldy, Waterman-Truell, Lloyd-Berry, and Ye-Ding, respectively.

Kawahara et al:: Antiplane shear wave scattering by cavities 


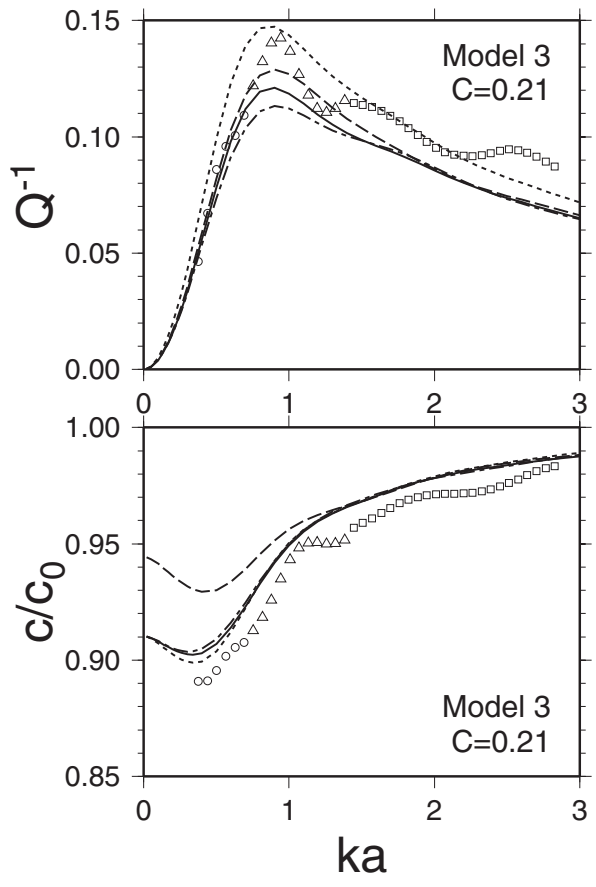

FIG. 6. Attenuation and dispersion curves for model 3. The open symbols denote the experimental values. The solid, dashed, dotted, and dash-dotted lines denote the predictions by the theories of Foldy, Waterman-Truell, Lloyd-Berry, and Ye-Ding, respectively.

which lie those of Foldy formula; any of these values are consistent with the experimental ones. In contrast, the Waterman-Truell formula (4) obviously disagrees with the other three as well as the experimental results. As $k a$ increases, however, the four theoretical values converge and equally follow the experimental ones. Concerning $Q^{-1}$, the formulas of Foldy, Waterman-Truell, and Ye-Ding give relatively similar values, whereas those by the Lloyd-Berry formula are somewhat larger. The experimental curve is located between the Waterman-Truell and Ye-Ding curves in the range $k a<1$. As $k a$ increases, it begins to corrugate, and approaches and slightly exceeds the Lloyd-Berry curve.

The oscillating behavior of the experimental $Q^{-1}$ curve is magnified in the case of a denser cavity distribution of model 3 ( $C=0.21$; Fig. 6). In this case, the experimental $Q^{-1}$ values considerably exceed all of the theoretical ones beyond $k a \sim 2$. Oscillating behavior is clearly observed also on the experimental dispersion curve. Here the experimental $c$ values are systematically smaller than any theoretical ones for any wavenumber considered.

Another noticeable feature of Figs. 5 and 6 is that the dispersion curves of the Foldy, Lloyd-Berry, and Ye-Ding formulas are nearly identical even in such cases of rather high concentrations, from which that of the Waterman-Truell is considerably deviated. The exceptional behavior of the latter might be due to the error in the Waterman-Truell theory claimed by Lloyd and Berry ${ }^{8}$ and also Linton and Martin. ${ }^{28,30}$ It would be worth mentioning that $\mathrm{Ye}^{40}$ demonstrated that the Waterman-Truell formula does not satisfy the Kramers-Kronig relation. This might be also attributed to the incorrectness of the formula.

In summary, it is reconfirmed that the Foldy theory predicts the attenuation and dispersion very accurately for suf-

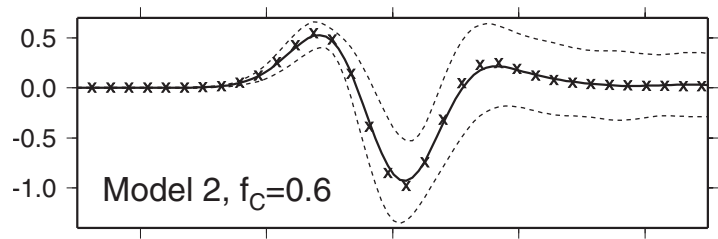

$\begin{array}{lllll}8 & 9 & 10 & 11 & 12\end{array}$

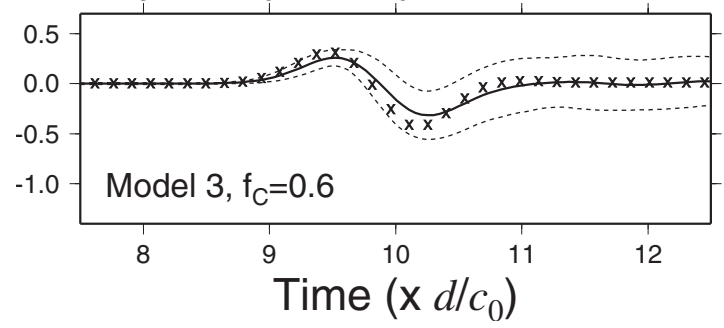

FIG. 7. The primary parts of the synthetic mean waves for models 2 and 3. The solid line and the two dotted ones denote the average and the standard deviation of the waveforms. The crosses indicate the mean waves predicted by the Foldy formula. Here $f_{C}=0.6$, for which the discrepancies between the synthetic and predicted mean waves are most remarkable in these models.

ficiently low cavity concentrations (say, $C \sim 0.02$ ). For $C$ $\sim 0.1$ and higher, the discrepancies between the Foldy predictions and experimental results are no more insignificant and are larger for the larger $C$, as may also be expected. In such situations, however, the other three theories also appear to be equally unsuccessful in describing the experimental results, despite the claim that they are corrected versions of the classical Foldy theory. None of the theories closely follows the experimental results on attenuation throughout the wavenumber range considered $(0.5<k a<3)$. Concerning dispersion, the Waterman-Truell theory obviously disagrees with the experiments even for $C \sim 0.1$; the other theories are consistent with the experiments for this concentration, but beyond it all of them systematically underestimate the magnitude of dispersion. Therefore it is hard to place one over the other, except for that the Foldy formula has the advantage of its high simplicity. Roughly speaking, the formula may be useful even for $C \sim 0.1$ if the relative error tolerance is as high as, say, $10 \%$. Indeed, the discrepancies between the synthetic mean waveforms and those predicted by the Foldy formula remain relatively small even for such a case, as demonstrated in Fig. 7(a). Figure 7(b) denotes, however, that it is not the case for $C \sim 0.2$ (model 3 ).

As the distributions become denser, the experimental attenuation and dispersion curves show the oscillating (or resonant) behavior more clearly. None of the present theories explains this behavior. This does not indicate the resonance of one cavity which could occur even without the others; otherwise, similar oscillation would be always observed on the theoretical curves, which are obtained from the scattering amplitude of an isolated scatterer. It is unlikely to be due to the computational errors because of the care taken to assure the accuracy of the simulations, as mentioned in Sec. III. It may not be also related to the finiteness of the scatterer distribution areas that is not assumed in the theories because the "resonant wavelengths" are apparently not correlated to the area size. For example, the first and second peaks above $k a$ $=1$ of the $Q^{-1}$ curve in Fig. 6 occur at around $k a=1.4$ and 2.5 , which correspond to the wavelengths $4.5 a$ and $2.5 a$, 
respectively, much smaller than $L^{\prime}=9.5 d=19 a$. Since all of the theories are based on the point scatterer assumption as stated in Sec. II, the behavior might be attributed to the effect of finite scatterer sizes (more accurately, finite exclusive areas of scatterers), which would be stronger for denser scatterer distributions. Adopting a pair-correlation function properly describing the cavity distributions would be necessary to recover the agreement with the experiments. This inference may be supported by a theoretical result of Varadan et al. ${ }^{11} \mathrm{In}$ order to make comparison with a laboratory-experimental result, they derived dispersion curves for lead spheres of a finite size embedded in an epoxy matrix, using the quasicrystalline approximation and a realistic pair-correlation function. Their results were given for two cases with $C$ $=0.05$ and 0.15 , and the dispersion curve for the latter case shows oscillation much more noticeably than the former. Nevertheless, the oscillating behavior is observed even in Fig. $4(2<k a<3)$, though slightly. This implies that the effect of finite scatterer sizes may not necessarily be negligible even for such a small $C$.

The validity of the theories has been discussed in terms of $C=\pi n a^{2}$ for a specific type of scatterers (cavities) so far. From a general viewpoint, however, it would be also important to consider the effect of the scattering strength, which would depend on not only $n$ but also the scattering amplitude of each scatterer $f(\theta)$. A possible measure of the strength might be $\left|n f(\theta) / k^{2}\right|{ }^{7}$, which seems to be assumed to be small, either explicitly or implicitly, in the present theories. As mentioned in Sec. III, the maximum value of $\left|n f(\theta) / k^{2}\right|$ for each model is given in Table I. It turns out that this value is fairly close to $C(\approx 0.75 C)$ in the present cases. For model 3 for which any theory does not work, it is 0.16 and actually not so small compared with unity. In addition, if one assumes weak scattering

$$
\left|n f(\theta) / k^{2}\right| \ll 1,
$$

then Foldy formula (3) yields

$$
\left|K^{2} / k^{2}-1\right| \ll 1,
$$

that is, $K$ is very close to $k$. Under this condition, both $Q^{-1}$ and $\left|c / c_{0}-1\right|$, defined by Eqs. (11) and (13), respectively, are equally of the order of $\left|n f(\theta) / k^{2}\right|$. Hence these two quantities may also work as approximate measures of the scattering strength; if the actual values of $Q^{-1}$ and $\left|c / c_{0}-1\right|$ were not so small as compared with unity, the theories would not reproduce their accurate values. This inference seems to be consistent with the present results; the maximum value of either $Q^{-1}$ or $\left|c / c_{0}-1\right|$ exceeds 0.1 for model 3 (Fig. 6), like $\max \left|n f(\theta) / k^{2}\right|$ in this case. More convincing discussions would require further similar experiments on different types of scatterers. Another possible factor limiting the validity of the theories might be the propagation distance $L^{\prime}$, which was chosen not in a systematic manner in the present study. Loosely speaking, only cases of relatively short distance were treated here. It would be of interest to confirm whether the present theories remain valid for much more larger distance, though it would require the significant increase in computational costs.

\section{CONCLUSION}

In this article, the attenuation and phase velocities of antiplane shear waves due to scattering by $2 \mathrm{D}$ cavities were experimentally measured, using computer simulations based on the boundary integral method of Benites et al. ${ }^{24}$ The results are then compared with the predictions by the classical Foldy theory and its three corrected versions: the theories of Waterman-Truell, Lloyd-Berry, and Ye-Ding. As long as the cavity concentrations remain small, the differences among the theoretical predictions are insignificant and any one is consistent with the experimental results. For higher concentrations (say, over 0.1), those differences become not negligible. In such situations, however, the experimental results show complicated dependence on wavenumbers, and none of the theoretical predictions matches the experimental results consistently. For example, all of the theories systematically underestimate $Q^{-1}$ for $2<k a<3$ and $\Delta c / c_{0}$ for any $k a$, respectively. Nevertheless, even the simple Foldy formula seems to be useful for concentrations up to about 0.1 if the error tolerance is relatively high.

Future work should deal with other types of scatterers from the practical viewpoint, such as inclusions either harder or softer than the matrix. This would be also helpful to clarify the validity of multiple scattering theories in terms of scattering strength. Researches focusing on the dependence of the attenuation and dispersion on the wave propagation distance would be also valuable.

\section{ACKNOWLEDGMENTS}

The authors appreciate Dr. Rafael Benites for providing us with detailed information on his previous works. Discussions with Dr. Haruo Sato were helpful in the early stage of this study. The critical comments of two anonymous reviewers are valuable for improving the manuscript. The figures in this paper were generated using the GMT software of Wessel and Smith. ${ }^{41}$ This study was supported partially by the Earthquake Research Institute Cooperative Research Program (1996-B-08, 2000-B-07, and 2001-B-02) and KAKENHI (11740254).

\footnotetext{
${ }^{1}$ A. Ishimaru, Wave Propagation and Scattering in Random Media (Academic, New York, 1978).

${ }^{2}$ S. M. Nair, D. K. Hsu, and J. H. Rose, "Porosity estimation using the frequency dependence of the ultrasonic attenuation," J. Nondestruct. Eval. 8, 13-26 (1989).

${ }^{3}$ H. Sato and M. C. Fehler, Seismic Wave Propagation and Scattering in the Heterogeneous Earth (Springer-Verlag, New York, 1998).

${ }^{4}$ L. L. Foldy, "The multiple scattering of waves. I. General theory of isotropic scattering by randomly distributed scatterers," Phys. Rev. 67, 107119 (1945).

${ }^{5}$ M. Lax, "Multiple scattering of waves," Rev. Mod. Phys. 23, 287-310 (1951).

${ }^{6}$ M. Lax, "Multiple scattering of waves. II. The effective field in dense systems," Phys. Rev. 85, 621-629 (1952).

${ }^{7}$ P. C. Waterman and R. Truell, "Multiple scattering of waves," J. Math. Phys. 2, 512-537 (1961).

${ }^{8}$ P. Lloyd and M. V. Berry, "Wave propagation through an assembly of spheres IV. Relation between different multiple scattering theories," Proc. Phys. Soc. London 91, 678-688 (1967).

${ }^{9}$ V. Twersky, "Acoustic bulk parameters in distributions of pair-correlated scatterers," J. Acoust. Soc. Am. 64, 1710-1719 (1978).

${ }^{10}$ L. Tsang, J. A. Kong, and T. Habashy, "Multiple scattering of acoustic waves by random distribution of discrete spherical scatterers with the qua-
} 
sicrystalline and Percus-Yevick approximation,” J. Acoust. Soc. Am. 71, 552-558 (1982).

${ }^{11} \mathrm{~V}$. K. Varadan, Y. Ma, and V. V. Varadan, "Scattering and attenuation of elastic waves in random media," Pure Appl. Geophys. 131, 577-603 (1989).

${ }^{12}$ F. V. Meulen, G. Feuillard, O. B. Matar, and F. Levassort, "Theoretical and experimental study of the influence of the particle size distribution on acoustic wave properties of strong inhomogeneous media," J. Acoust. Soc. Am. 110, 2301-2307 (2001).

${ }^{13} \mathrm{Z}$. Ye and L. Ding, "Acoustic dispersion and attenuation relations in bubbly mixture," J. Acoust. Soc. Am. 98, 1629-1636 (1995).

${ }^{14} \mathrm{~F}$. S. Henyey, "Corrections to Foldy's effective medium theory for propagation in bubble clouds and other collections of very small scatterers," J Acoust. Soc. Am. 105, 2149-2154 (1999).

${ }^{15}$ R.-B. Yang, "A dynamic generalized self-consistent model for wave propagation in particulate composites," J. Appl. Mech. 70, 575-582 (2003).

${ }^{16}$ D. G. Aggelis, S. V. Tsinopoulos, and D. Polyzos, "An iterative effective medium approximation (IEMA) for wave dispersion and attenuation predictions in particulate composites, suspensions and emulsions," J. Acoust. Soc. Am. 116, 3443-3452 (2004).

${ }^{17}$ J. Mobley, K. R. Waters, C. S. Hall, and J. N. Marsh, "Measurements and predictions of the phase velocity and attenuation coefficient in suspensions of elastic microsphere," J. Acoust. Soc. Am. 106, 652-659 (1999).

${ }^{18} \mathrm{D}$. J. McClements, "Comparison of multiple scattering theories with experimental measurements in emulsions," J. Acoust. Soc. Am. 91, 849-853 (1992).

${ }^{19}$ A. K. Holmes, R. E. Challis, and D. J. Wedlock, "A wide bandwidth study of ultrasound velocity and attenuation in suspensions: Comparison of theory with experimental measurements," J. Colloid Interface Sci. 156, 261-268 (1993).

${ }^{20}$ A. K. Hipp, G. Storti, and M. Morbidelli, "On multiple-particle effects in the acoustic characterization of colloidal dispersions," J. Phys. D: Appl. Phys. 32, 568-576 (1999).

${ }^{21}$ C. Layman, N. S. Murthy, R.-B. Yang, and J. Wu, "The interaction of ultrasound with particulate composites," J. Acoust. Soc. Am. 119, 1449 1456 (2006).

${ }^{22}$ A. Richter, F. Babick, and S. Ripperger, "Polydisperse particle size characterization by ultrasonic attenuation spectroscopy for systems of diverse acoustic contrast in the large particle limit," J. Acoust. Soc. Am. 118, 1394-1405 (2005).

${ }^{23}$ M.-F. Poujol-Pfefer, "Application of an homogenization model to the acoustical propagation in inhomogeneous media," J. Sound Vib. 184, 665679 (1995).

${ }^{24}$ R. Benites, K. Aki, and K. Yomogida, "Multiple scattering of SH waves in 2-D media with many cavities," Pure Appl. Geophys. 138, 353-390
(1992).

${ }^{25}$ S. Biwa, S. Yamamoto, F. Kobayashi, and N. Ohno, "Computational multiple scattering analysis for shear wave propagation in unidirectional composites," Int. J. Solids Struct. 41, 435-457 (2004).

${ }^{26}$ T.-K. Hong and B. L. N. Kennett, "Scattering of elastic waves in media with a random distribution of fluid-filled cavities: Theory and numerical modelling," Geophys. J. Int. 159, 961-977 (2004).

${ }^{27}$ Y. Suzuki, J. Kawahara, T. Okamoto, and K. Miyashita, "Simulations of SH wave scattering due to cracks by the 2-D finite difference method," Earth, Planets Space 58, 555-567 (2006).

${ }^{28}$ C. M. Linton and P. A. Martin, "Multiple scattering by random configurations of circular cylinders: Second-order corrections for the effective wavenumber," J. Acoust. Soc. Am. 117, 3413-3423 (2005).

${ }^{29}$ Y. C. Angel and C. Aristégui, "Analysis of sound propagation in a fluid through a screen of scatterers," J. Acoust. Soc. Am. 118, 72-82 (2005).

${ }^{30}$ C. M. Linton and P. A. Martin, "Multiple scattering by multiple spheres: A new proof of the Lloyd-Berry formula for the effective wavenumber," SIAM J. Appl. Math. 66, 1649-1668 (2006).

${ }^{31}$ Y.-H. Pao and C.-C. Mow, Diffraction of Elastic Waves and Dynamic Stress Concentrations (Crane Russak, New York, 1973).

${ }^{32} \mathrm{~L}$. G. Copley, "Integral equation method for radiation from vibrating bodies," J. Acoust. Soc. Am. 41, 807-816 (1967).

${ }^{33}$ F. J. Sánchez-Sesma and E. Rosenblueth, "Ground motion at canyons of arbitrary shape under incident SH waves," Earthquake Eng. Struct. Dyn. 7, 441-450 (1979).

${ }^{34}$ M. Dravinski, "Ground motion amplification due to elastic inclusions in a half-space," Earthquake Eng. Struct. Dyn. 11, 313-335 (1983).

${ }^{35}$ G. D. Manolis and D. E. Beskos, Boundary Element Methods in Elastodynamics (Unwin Hyman, London, 1988).

${ }^{36} \mathrm{R}$. Benites and K. Aki, "Boundary integral-Gaussian beam method for seismic wave scattering: SH waves in two-dimensional media," J. Acoust. Soc. Am. 86, 375-386 (1989).

${ }^{37}$ T. Pointer, E. Liu, and J. A. Hudson, "Numerical modelling of seismic waves scattered by hydrofractures: Application of the indirect boundary element method," Geophys. J. Int. 135, 289-303 (1998).

${ }^{38}$ Wave Analysis and Boundary Element Methods, edited by S. Kobayashi (Kyoto University Press, Kyoto, 2000), in Japanese.

${ }^{39}$ J. M. Martin and S. M. Flatté, "Intensity images and statistics from numerical simulation of wave propagation in 3-D random media," Appl. Opt. 27, 2111-2126 (1988).

${ }^{40} \mathrm{Z}$. Ye, "Acoustic dispersion and attenuation in many spherical scatterer systems and the Kramers-Kronig relations," J. Acoust. Soc. Am. 101, 3299-3305 (1997).

${ }^{41}$ P. Wessel and W. H. F. Smith, "New, improved version of generic mapping tools released," EOS Trans. Am. Geophys. Union 79, 579 (1998). 